\title{
ON CONFORMALLY RECURRENT SPACES OF SECOND ORDER
}

\author{
M. C. CHAKI and A. N. ROY CHOWDHURY
}

(Received 6 December 1967)

\section{Introduction}

In a recent paper [ 1 ] Adati and Miyazawa studied conformally recurrent spaces, that is, Riemannian spaces defined by $C_{i j k, l}^{h}=\lambda_{l} C_{i j k}^{h}$ where $C_{i j k}^{h}$ is the conformal curvature tensor:

$$
\begin{gathered}
C_{i j k}^{h}=R_{i j k}^{h}-\frac{1}{n-2}\left(R_{k}^{h} g_{i j}-R_{j}^{h} g_{i k}+R_{i j} \delta_{k}^{h}-R_{i k} \delta_{j}^{h}\right) \\
+\frac{R}{(n-1)(n-2)}\left(\delta_{k}^{h} g_{i j}-\delta_{j}^{h} g_{i k}\right),
\end{gathered}
$$

$\lambda_{l}$ is a non-zero vector and comma denotes covariant differentiation with respect to the metric tensor $g_{i j}$. The present paper is concerned with nonflat Riemannian spaces $V_{n}(n>3)$ defined by

$$
C_{i j k, l m}^{h}=a_{l m} C_{i j k}^{h}
$$

where $a_{l m}$ is a tensor not identically zero. We shall call a Riemannian space defined by (2) a conformally recurrent space of second order and shall denote an $n$-space of this kind by $C\left({ }^{2} K_{n}\right)$. A Riemannian space whose curvature tensor satisfies $R_{i j k, l m}^{h}=\bar{a}_{l m} R_{i j k}^{h}$ was called a Recurrent space of second order by A. Lichnerowicz [2]. Such an $n$-space shall be denoted by ${ }^{2} K_{n}$. Evidently every ${ }^{2} K_{n}$ is a $C\left({ }^{2} K_{n}\right)$ but the converse is not necessarily true. Sections 2 and 3 of this paper deal with Einstein and 2-Ricci-recurrent $C\left({ }^{2} K_{n}\right)$ respectively while section 4 deals with $C\left({ }^{2} K_{n}\right)$ admitting a parallel vector field. In the last section it will be shown that a Riemannian space satisfying $W_{i j k, l m}^{h}=a_{l m}^{\prime} W_{i j k}^{h}$ where $W_{i j k}^{h}$ is Weyl's projective curvature tensor is a $C\left({ }^{2} K_{n}\right)$.

\section{Tensor of recurrence in a $C\left({ }^{2} K_{n}\right)$}

We have

$$
\left(C_{h i j k} C^{h i j k}\right)_{, l m}=2 C_{h i j k l m} C^{h i j k}+2 C^{h i j k}{ }_{, l} C_{h i j k, m}
$$

Therefore, in a $C\left({ }^{2} K_{n}\right)$ 
Hence

$$
\left(C_{n i j k} C^{h i j k}\right)_{, l m}=2 a_{l m} C_{h i j k} C^{h i j k}+2 C^{h i j k}{ }_{, 2} C_{h j j k, m}
$$

So either

$$
2\left(a_{l m}-a_{m l}\right) C_{h i j k} C^{h i j k}=0
$$

(i) $C_{h i j k} C^{h i j k}=0$ or

(ii) $a_{\imath m}=a_{m l}$

If the space is of positive definite metric and not conformally flat, then (i) cannot hold and therefore $a_{l m}$ is symmetric.

Again, if in a $C\left({ }^{2} K_{n}\right), R_{i j}=0$, then from (1) and (2) it follows that $R_{i j k, l m}^{h}=a_{l m} R_{i j k}^{h}$, that is, the space is a ${ }^{2} K_{n}$. It is already known that for a ${ }^{2} K_{n}$ the tensor of recurrence is symmetric. Hence if for a $C\left({ }^{2} K_{n}\right), R_{i j}=0$, then its tensor of recurrence is symmetric. We can therefore state the following theorems:

THEOREM 1. If a $C\left({ }^{2} K_{n}\right)$ with positive definite metric is not conformally flat, then its tensor of recurrence is symmetric.

THEOREM 2. If for a $C\left({ }^{2} K_{n}\right)$ the Ricci tensor is a zero tensor, then its tensor of recurrence is symmetric.

\section{Einstein $C\left({ }^{2} K_{n}\right)$}

If a $C\left({ }^{2} K_{n}\right)$ is an Einstein space, defined by $R_{i j}=(R / n) g_{i j}$, then

$$
R_{i j, l m}=0
$$

Let us suppose that an Einstein $C\left({ }^{2} K_{n}\right)$ is a ${ }^{2} K_{n}$. Then $R_{i j k, l m}^{h}=d_{l m} R_{i j k}^{h}$ for a non-zero tensor $d_{l m}$. Consequently $R_{i j, l m}=d_{l m} R_{i j}$. Therefore in virtue of (2.1) $R_{i j}=0$, because $d_{l m} \neq 0$. Hence $R=0$.

Again, if in an Einstein $C\left({ }^{2} K_{n}\right), R=0$ then $R_{i j}=0$ and therefore the space is a ${ }^{2} K_{n}$. In an Einstein $C\left({ }^{2} K_{n}\right)$ of zero scalar curvature

$$
R_{i j k, l m}^{h}=a_{l m} R_{i j k}^{h}
$$

Making use of (2.2) and the Bianchi identity we get

$$
a_{l m} R_{i j k}^{h}+a_{j m} R_{i k l}^{h}+a_{k m} R_{i l j}^{h}=0
$$

Multiplying (2.3) by $a_{t}^{l}$ where $a_{t}^{l}=g^{l p} a_{p t}$ we have

$$
a_{t}^{l} a_{l m} R_{i j k}^{h}+a_{t}^{l} a_{j m} R_{i k l}^{h}+a_{t}^{l} a_{k m} R_{i l j}^{h}=0
$$

$R_{i j}=0$ implies $a_{h m} R_{i j k}^{h}=0$ by contracting $h$ and $k$ in (2.3). Hence $a_{m}^{t} R_{k i t}^{p}=0$. Using this (2.4) reduces to $a_{t}^{l} a_{l m} R_{i j k}^{h}=0$. Since the space is not flat, $a_{t}^{l} a_{l m}=0$. Thus we have the following theorems: 
THEOREM 3. A necessary and sufficient condition that an Einstein $C\left({ }^{2} K_{n}\right)$ may be a ${ }^{2} K_{n}$ is that its scalar curvature is zero.

TheOREM 4. In an Einstein $C\left({ }^{2} K_{n}\right)$ of zero scalar curvature $a_{t}^{l} a_{l m}=0$.

We now consider an Einstein $C\left({ }^{2} K_{n}\right)$ of non-zero scalar curvature.

From (2.1) as well as (1) and (2) we have

$$
R_{h i j k, l m}=a_{l m} T_{h i j k}
$$

where

$$
T_{h i j k}=R_{h i j k}-\frac{R}{n(n-1)}\left(g_{h k} g_{i j}-g_{h j} g_{i k}\right)
$$

Using (2.5) Walker's Lemma 1 [3], namely

$$
R_{h i j k, l m}-R_{h i j k, m l}+R_{j k l m, h i}-R_{j k l m, i h}+R_{l m h i, j k}-R_{l m h i, k j}=0
$$

reduces to

$$
b_{l m} T_{h i j k}+b_{h i} T_{j k l m}+b_{j k} T_{l m h i}=0
$$

where

$$
b_{l m}=a_{l m}-a_{m l} \text {. }
$$

Since $T_{h i j k}=T_{j k h i}$, by Walker's Lemma 2 [3] we have from (2.7) either $b_{l m}=0$ or $T_{h i j k}=0$. Hence we have the following theorem:

THEOREM 5. If a $C\left({ }^{2} K_{n}\right)$ is an Einstein space of non-zero scalar curvature, then either its tensor of recurrence is symmetric or it is a space of constant curvature.

\section{2-Ricci-recurrent $C\left({ }^{2} K_{n}\right)$}

In a previous paper [4] we called a non-flat Riemannian space a Ricci-recurrent space of second order, or briefly a 2-Ricci-recurrent space if its Ricci tensor satisfies

$$
R_{i j, k l}=a_{k l}^{*} R_{i j}
$$

and $R_{i j} \not \equiv 0$ for a non-zero tensor $a_{k l}^{*}$.

We put

$$
\Pi_{i j}=\frac{1}{n-2}\left[R_{i j}-\frac{R}{2(n-1)} g_{i j}\right]
$$

and

$$
D_{i j k}^{h}=\Pi_{k}^{h} g_{i j}-\Pi_{j}^{h} g_{i k}+\Pi_{i j} \delta_{k}^{h}-\Pi_{i k} \delta_{j}^{h}
$$

where $\Pi_{k}^{h}=g^{h t} \Pi_{t k}$. Then 


$$
C_{i j k}^{h}=R_{i j k}^{h}-D_{i j k}^{h}
$$

Moreover,

$$
\Pi=g^{i j} \Pi_{i j}=\frac{R}{2(n-1)}
$$

and

$$
D_{h i j k}=-D_{i h j k}=-D_{h i k j}=D_{j k h i}
$$

where $D_{h i j k}=g_{h t} D_{i j k}^{t}$. Let us suppose that $\Pi_{i j}$ is a non-zero tensor satisfying

$$
\Pi_{i j, k l}=a_{k l}^{*} \Pi_{i j}
$$

where $a_{k l}^{*}$ is a non-zero tensor. Then $\Pi_{, k l}=a_{k l}^{*} \Pi$ or,

$$
R_{, k l}=a_{k l}^{*} R
$$

From (3.2) we have

Therefore

$$
\Pi_{i j, k l}=\frac{1}{n-2}\left[R_{i j, k l}-\frac{R_{, k l}}{2(n-1)} g_{i j}\right] .
$$

$$
\frac{1}{n-2} R_{i j, k l}=a_{k l}^{*}\left[\Pi_{i j}+\frac{R}{2(n-1)(n-2)} g_{i j}\right]=a_{k l}^{*} \frac{1}{n-2} R_{i j}
$$

Hence

$$
R_{i j, k l}=a_{k l}^{*} R_{i j}
$$

Conversely, if (3.8) holds, then

$$
\Pi_{i j, k l}=a_{k l}^{*} \Pi_{i j}
$$

We can therefore state the following lemma:

Lemma. If in a Riemannian space, the tensor $\Pi_{i j}$, defined by (3.2), is a non-zero tensor, then the space is 2-Ricci-recurrent if and only if $\Pi_{i j, k l}=a_{k l}^{*} \Pi_{i j}$ for a non-zero tensor $a_{k l}^{*}$.

We now suppose that in a $C\left({ }^{2} K_{n}\right),(3.6)$ holds. Differentiating (3.3) covariantly we have

$$
D_{i j k, l m}^{h}=\Pi_{k, l m}^{h} g_{i j}-\Pi_{j, l m}^{h} g_{i k}+\Pi_{i j, l m} \delta_{k}^{h}-\Pi_{i k, l m} \delta_{j}^{h}
$$

Using (3.6) we get

$$
D_{i j k, l m}^{h}=a_{l m}^{*} D_{i j k}^{h}
$$

From (3.4) we have

$$
\begin{aligned}
R_{i j k, l m}^{h} & =C_{i j k, l m}^{h}+D_{i j k, l m}^{h} \\
& =a_{l m} C_{i j k}^{h}+a_{l m}^{*} D_{i j k}^{h}
\end{aligned}
$$


Therefore

$$
R_{h i j k, l m}-R_{h i j k, m l}=b_{l m} C_{h i j k}+c_{l m}^{\prime} D_{h i j k}
$$

where $b_{l m}$ is given by (2.8) and $c_{l m}^{\prime}=a_{l m}^{*}-a_{m l}^{*}$. Now using (3.10) Walker's lemma (2.6) can be written as

$$
\begin{aligned}
b_{l m} C_{h i j k}+b_{h i} C_{j k l m} & +b_{j k} C_{l m h i}+c_{l m}^{\prime} D_{h i j k} \\
& +c_{h i}^{\prime} D_{j k l m}+c_{j k}^{\prime} D_{l m h i}=0 .
\end{aligned}
$$

Let us suppose that $a_{l m}$ is symmetric. Then $b_{l m}=0$. Hence (3.11) reduces to

$$
c_{l m}^{\prime} D_{h i j k}+c_{h i}^{\prime} D_{j k l m}+c_{j k}^{\prime} D_{l m h i}=0 \text {. }
$$

Since

$$
C_{i j}=0, \quad \Pi_{i j}=\frac{1}{n-2}\left[D_{i j}-\frac{D}{2(n-1)} g_{i j}\right] .
$$

Hence $\Pi_{i j} \neq 0$ implies $D_{i j k l} \neq 0$. Also $D_{h i j k}=D_{j k h i}$. Hence applying Walker's Lemma 2 to (3.12) we have $c_{l m}^{\prime}=0$. Hence $a_{l m}^{*}$ is symmetric. Next, we suppose that $a_{l m}^{*}$ is symmetric. Then $c_{l m}^{\prime}=0$ and it follows from (3.11) that

$$
b_{l m} C_{h i j k}+b_{h i} C_{j k l m}+b_{j k} C_{l m h i}=0 .
$$

Hence if $C_{h i j k} \neq 0$, it follows from (3.13) that $b_{l m}=0$ whence $a_{l m}$ is symmetric.

We can therefore state the following theorems:

THEOREM 6. If a $C\left({ }^{2} K_{n}\right)$ which is not conformally flat is a 2-Riccirecurrent space, then its tensor of recurrence is symmetric if and only if the tensor of 2-Ricci-recurrence is symmetric.

THEOREM 7. If a $C\left({ }^{2} K_{n}\right)$ is a 2-Ricci-recurrent space, then the tensor of 2-Ricci-recurrence is symmetric if the tensor of recurrence of $C\left({ }^{2} K_{n}\right)$ is so.

\section{4. $C\left({ }^{2} K_{n}\right)$ admitting a parallel vector field}

Let us assume that there exists a parallel vector field $v^{i}$ in a $C\left({ }^{2} K_{n}\right)$. Then $v^{i}, l=0$. Therefore $v^{i},{ }_{l m}-v^{i}, m l=0$. Hence using the Ricci identity and the Bianchi identity we have

Therefore,

$$
\begin{aligned}
v^{t} R_{t l m}^{h} & =0, & v^{t} R_{t l} & =0 \\
v^{t} R_{t l m, n}^{h} & =0, & v^{t} R_{t l, n} & =0 .
\end{aligned}
$$

$$
v^{t} R_{i j k, t}^{h}=0, \quad v^{t} R_{i j, t}=0, \quad v^{t} R_{, t}=0
$$


From (2) we have $v^{l} C_{i j k, l m}^{h}=v^{l} a_{l m} C_{i j k}^{h}$ or,

$$
\begin{array}{r}
v^{i}\left[R_{i j k, l m}^{h}-\frac{1}{n-2}\left(R_{k, l m}^{h} g_{i j}-R_{j, l m}^{h} g_{i k}+R_{i j, l m} \delta_{k}^{h}-R_{i k, l m} \delta_{j}^{h}\right)\right. \\
\left.+\frac{1}{(n-1)(n-2)} R_{, l m}\left(\delta_{k}^{h} g_{i j}-\delta_{j}^{h} g_{i k}\right)\right]=v^{l} a_{l m} C_{i j k}^{n} .
\end{array}
$$

Using (4.1) the left hand side of (4.2) reduces to zero. Hence $v^{l} a_{l m} C_{i j k}^{h}=0$. Thus we have the following theorem:

THEOREM 8. If a $C\left({ }^{2} K_{n}\right)$ admits a parallel vector field $v^{i}$, then either the space is conformally flat or $v^{2} a_{l m}=0$.

\section{Projective recurrent spaces of second order}

A. Riemannian space $V_{n}(n \geqq 3)$ satisfying

$$
W_{i j k, l m}^{h}=a_{l m}^{\prime} W_{i j k}^{n}
$$

for a non-zero tensor $a_{l m}^{\prime}$ where $W_{i j k}^{h}$ is Weyl's projective curvature tensor

$$
W_{i j k}^{h}=R_{i j k}^{h}-\frac{1}{n-1}\left(\delta_{k}^{h} R_{i j}-\delta_{j}^{h} R_{i k}\right)
$$

shall be called a projective recurrent space of second order.

From (5.2) we have

$$
W_{i j k, l m}^{h}=R_{i j k, l m}^{h}-\frac{1}{n-1}\left(\delta_{k}^{h} R_{i j, l m}-\delta_{j}^{h} R_{i k, l m}\right) .
$$

Substituting (5.2) and (5.3) in (5.1) we get

$$
\begin{aligned}
R_{i j k, l m}^{h}-\frac{1}{n-1}\left(\delta_{k}^{h} R_{i j, l m}-\delta_{j}^{h} R_{i k, l m}\right) & \\
=a_{l m}^{\prime} & {\left[R_{i j k}^{h}-\frac{1}{n-1}\left(\delta_{k}^{h} R_{i j}-\delta_{i}^{h} R_{i k}\right)\right] . }
\end{aligned}
$$

Therefore,

$$
R_{k, l m}^{h}=a_{l m}^{\prime} R_{k}^{h}+\frac{1}{n}\left(R_{, l m}-a_{l m}^{\prime} R\right) \delta_{k}^{h}
$$

and

$$
R_{i j, l m}=a_{l m}^{\prime} R_{i j}+\frac{1}{n}\left(R_{, l m}-a_{l m}^{\prime} R\right) g_{i j}
$$


Again from (1) we have

$$
\begin{aligned}
C_{i j k, l m}^{h}= & R_{i j k, l m}^{h}-\frac{1}{n-2}\left(R_{k, l m}^{h} g_{i j}-R_{j, l m}^{h} g_{i k}\right. \\
& \left.+R_{i j, l m} \delta_{k}^{h}-R_{i k, l m} \delta_{j}^{h}\right)+\frac{1}{(n-1)(n-2)} R_{, l m}\left(\delta_{k}^{h} g_{i j}-\delta_{j}^{h} g_{i k}\right) .
\end{aligned}
$$

Making use of (5.4), (5.5) and (5.6) we have from (5.7)

$$
C_{i j k, l m}^{h}=a_{l m}^{\prime} C_{i j k}^{h} \text {. }
$$

Hence we have the following theorem:

THEOREM 9. Every $n$-dimensional $(n>3)$ projective recurrent space of second order is a $C\left({ }^{2} K_{n}\right)$.

\section{References}

[1] T. Adati and T. Miyazawa, 'On a Riemannian space with recurrent conformal curvature', Tensor N.S., 18 (1967), 348-354.

[2] A. Lichnerowicz, 'Courbure, nombres de Betti, et espaces symétriques', Proc. Int. Cong. of Math. 2 (1960), 216-222.

[3] A. G. Walker, 'On Ruse's spaces of recurrent curvature', Proc. Lond. Math. Soc. (2) 52 (1950), 36-34.

[4] M. C. Chaki and A. N. Roy Chowdhury, 'On Ricci recurrent spaces of Second Order', Indian. J. Math. 9 (1967).

Department of Pure Mathematics

University of Calcutta

and

Department of Mathematics

Durgapur Engineering College 